\title{
Plastid mRNAs are neither spliced nor edited in maize and cauliflower mitochondrial in organello systems
}

\author{
NINA BOLLE, INGA HINRICHSEN, and FRANK KEMPKEN \\ Abteilung Botanische Genetik und Molekularbiologie, Botanisches Institut und Botanischer Garten, Christian-Albrechts-Universität zu Kiel, \\ 24098 Kiel, Germany
}

\begin{abstract}
The process of RNA editing in chloroplasts and higher plant mitochondria displays some similarities, raising the question of common or similar components in editing apparatus of these two organelles. To investigate the ability of plant mitochondria to edit plastid transcripts, we employed a previously established mitochondrial maize and cauliflower in organello system. Two plastid genes, Zea mays ndhB and ycf 3 containing group II introns and several editing sites, were introduced into mitochondria. The genes were transcribed in organello. However, these transcripts of the plastid genes are neither spliced nor edited in plant mitochondria. A comparison of maize $n d h B$ editing sites and maize mitochondrial editing sites reveals considerable sequence similarities between three $n d h B$ editing sites and several mitochondrial sites. Nevertheless, these ndhB editing sites were not recognized in the mitochondria. Thus, we present for the first time direct evidence that the factors present in the plant mitochondria are not sufficient to allow editing and splicing of plastid transcripts.
\end{abstract}

Keywords: RNA editing; RNA splicing; mitochondria in organello; ndhB; ycf3

\section{INTRODUCTION}

Transcripts of higher plant organelles are subject to various processing events, including RNA editing. This process is characterized by C-to- $\mathrm{U}$ and rare U-to-C conversions in transcripts present in plant mitochondria and chloroplasts (for review, see Shikanai 2006). The extent of RNA editing differs greatly between the organelles from up to 500 editing sites in plant mitochondria (summarized in Mower and Palmer 2006) and $\sim 30$ editing sites in chloroplasts (summarized in Zeng et al. 2007). While pentatricopeptide repeat $(\mathrm{PPR})$ proteins are known to be involved in editing site selection in plastids (Kotera et al. 2005; Okuda et al. 2006, 2007), factors binding the cis-elements of mitochondrial RNA editing sites have not yet been identified. However, we have recently presented data that support a self-guiding-transcript model for RNA editing in mitochondria of higher plants, indicating that such factors may not be needed (Bolle and Kempken 2006). In this model the editing information is contained in the transcript itself, and

Reprint requests to: Frank Kempken, Abteilung Botanische Genetik und Molekularbiologie, Botanisches Institut und Botanischer Garten, Christian-Albrechts-Universität zu Kiel, Olshausenstraße 40, 24098 Kiel, Germany; e-mail: fkempken@bot.uni-kiel.de; fax: +49-431-880-4248.

Article published online ahead of print. Article and publication date are at http://www.rnajournal.org/cgi/doi/10.1261/rna.758307. the ability to edit editing sites would be based on the structural formation of the RNA transcript.

The finding of sequence similarities between editing sites of plant mitochondria and chloroplasts (Maier et al. 1992) led to the question whether both plant organelles have a common RNA editing mechanism and components. However, despite these similarities, a plant mitochondrial sequence was shown not to be edited in transgenic chloroplasts (Sutton et al. 1995). Zeltz et al. (1996) later analyzed the RNA editing of a rice rpoB transcript transferred from chloroplast to mitochondrial genome during evolution, and found all editing sites to remain unedited. Up to now, no transformation system for plant mitochondria has been established. Therefore, we set out to use the previously established mitochondrial in organello editing systems (Staudinger and Kempken 2003; Bolle and Kempken 2006) to investigate the ability of plant mitochondria to recognize and edit plastid transcripts. In the previous studies we have shown that transcripts from introduced Arabidopsis thaliana cox 2 and Zea mays cox 2 were efficiently spliced and edited in the heterologous maize and cauliflower mitochondria. Using these in organello systems we are able to provide here the first direct evidence that plastid group II introns are not spliced from RNAs in isolated plant mitochondria nor is there any evidence for RNA editing. These results are discussed with respect to recent data 
regarding the mechanism of RNA editing site recognition in plant mitochondria (Bolle and Kempken 2006).

\section{RESULTS AND DISCUSSION}

To investigate whether plastid transcripts are spliced and edited in plant mitochondria, we chose the coding sequences of $n d h \mathrm{~B}$ and $y c f 3$ including intronic sequences from $Z$. mays. The $n d h \mathrm{~B}$ and $y c f 3$ open reading frames (ORFs) contain six and two RNA editing sites in the coding sequences, respectively, which were shown to be fully edited in maize plastids (Peeters and Hanson 2002). In addition, both plastid genes contain group II introns: One is present in the $n d h \mathrm{~B}$ ORF and two in the $y c f 3$ ORF (Fig. 1A). To ensure transcription of the plastid sequences, they were cloned under the control of mitochondrial regulatory sequences. Both plastid sequences were fused with the $5^{\prime}$ and $3^{\prime}$ noncoding sequences of the $Z$. mays atp 6 gene. To examine the potential influence of the noncoding sequences on RNA editing and splicing, we additionally constructed vector pNB569 containing $A$. thaliana cox2 regulatory sequences fused with the $n d h \mathrm{~B}$ sequence (Fig. 1A).

Using the previously established in organello systems with maize (Staudinger and Kempken 2003) and cauliflower mitochondria (Bolle and Kempken 2006), the general ability of the mitochondria to process mRNAs was tested with mitochondrial $A$. thaliana and $Z$. mays cox 2 constructs. In all cases, we observed full RNA processing of cox2 transcripts, i.e., splicing and editing (not shown) proving the mitochondria being fully active.

Chimeric $n d h \mathrm{~B}$ and $y c f 3$ constructs were electroporated into the isolated mitochondria from maize and cauliflower, in organello incubation was performed, and RNA was isolated from the mitochondria. DNase I treatment was performed to remove residual DNA, which was tested by control PCR reactions (not shown). Using the oligonucleotide pairs shown in Figure 1A, specific amplification of the chimeric transcripts was achieved. However, plant mitochondrial genomes contain sequences of plastid origin, e.g., the $n d h \mathrm{~B}$ gene in Z. mays (Stern and Lonsdale 1982; Clifton et al. 2004). Hence, to exclude the possibility that RT-PCR amplicons are derived from those promiscuous plastid sequences or from contaminating plastid RNA, RT-PCR was performed using the specific primer pairs with RNA from untreated mitochondria (not shown). None of the primer pairs yielded products in the RT-PCR, showing that they are applicable for specific amplification of the chimeric transcripts. RT-PCR was performed with RNAs from the electroporated maize and cauliflower mitochondria as templates, and cDNA of $n d h \mathrm{~B}$ and $y c f 3$ transcripts was obtained (see Fig. 1B). As indicated by the size of the cDNA, and confirmed by sequencing of these fragments, transcripts of all three constructs were obtained, i.e., none of them were spliced. Since group II introns require for efficient in vivo splicing intron-encoded maturases or host- a

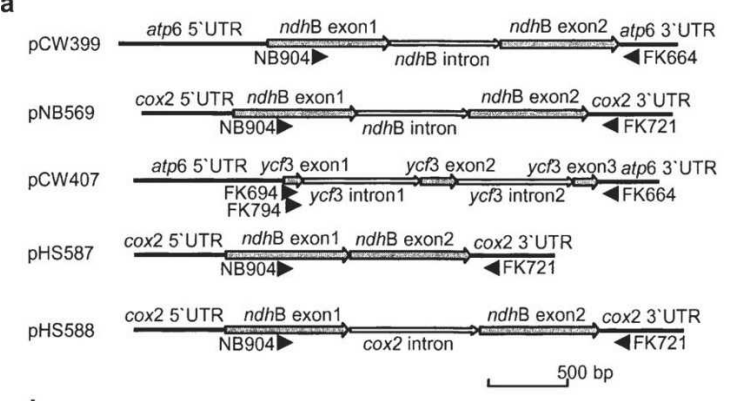

b
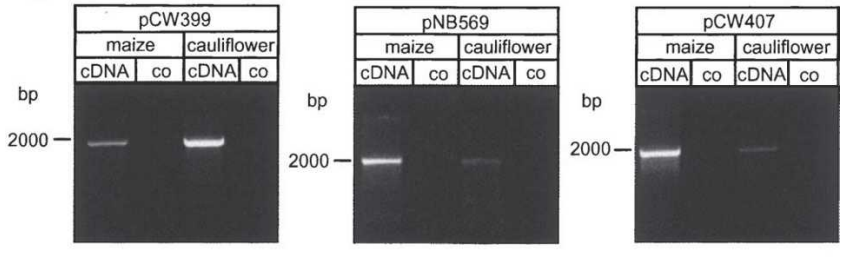

c
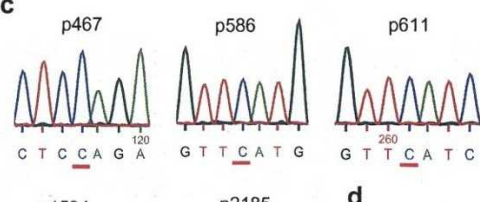

p737

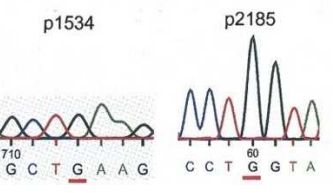

d $p 45$
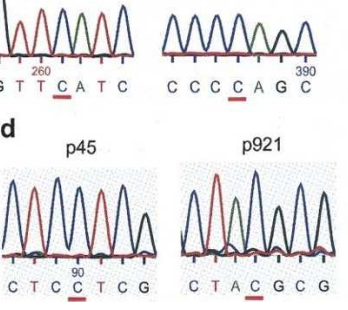

FIGURE 1. (A) Maps of the constructs pCW399, pNB569, pCW407, pHS587, and pHS588. The Z. mays atp6 and A. thaliana cox2 $5^{\prime}$ and 3' untranslated regions (UTR) are shown as black lines. The gray and white arrows indicate the exon-intron structure of the cloned coding regions of the $n d h \mathrm{~B}$ gene and $y c f 3$ gene from $Z$. mays. The construct pHS588 contains the intron from the cox 2 gene of $Z$. mays. The locations of oligonucleotides are indicated by black arrow heads. (B) RT-PCR amplifications from in organello assays with maize and cauliflower mitochondria electroporated with the chimeric constructs pCW399, pNB569, or pCW407. RT-PCR was performed using the specific oligonucleotide pairs NB904 (5'-CAACTTTATGTATTCCTC TATCCGTAG-3')/FK664 (5'-GCTACACCTAATTCCAGACC-3') for transcripts of pCW399, NB904/FK721 (5'-GCGAGCGAGTTAAG CCTCTA-3') for transcripts of pNB569 and for transcripts of pCW407 FK694 (5'-CCCGGGATGCCTAGATCC-3')/FK664 with maize and FK794 (5'-GGAAATTTCATTGATAAGACCTC-3')/FK664 with cauliflower mitochondrial RNA. co, RT-PCR control. $(C, D)$ Sequence analysis of RT-PCR products from in organello assays. RTPCR amplicons were sequenced directly or cloned before sequencing in the case of $y c f 3$ cDNA. Exemplified chromatograms are shown here: $(C) n d h \mathrm{~B}$ editing sites from transcripts of pCW399 (introduced into maize mitochondria). (D) $y c f 3$ editing sites from transcripts of pCW407 (introduced into cauliflower mitochondria). The chromatograms show that none of the $n d h \mathrm{~B}$ or $y c f 3$ editing sites were edited in the chimeric transcripts. The editing sites are numbered according to their position in the ORF and underlined.

encoded splicing factors, this result indicates that mitochondrial localized splicing factors are specific for mitochondrial introns and therefore nonfunctional in plastid introns. In the last few years, multiple protein factors required for organellar RNA splicing of group II introns have 
been identified. For example, in maize chloroplasts, three protein complexes were identified, required for splicing of different subsets of group II introns (Jenkins et al. 1997; Jenkins and Barkan 2001; Ostheimer et al. 2003). It was shown that splicing of the $n d h \mathrm{~B}$ intron in maize chloroplasts needs the two nuclear-encoded splicing factors CRS2 and CAF2, whereas splicing of the first intron of maize $y c f 3$ requires CRS2, CAF1, and CAF2. CRS2 seems to be a chloroplast-specific protein, since it can be imported into and is enriched in isolated chloroplasts (Jenkins and Barkan 2001). The result of plant mitochondria not being capable to splice plastid transcripts is in contrast to the appropriate inverse study from Herdenberger et al. (1994), where a mitochondrial intron was reported to be spliced in algal chloroplasts.

Furthermore, sequence analysis of the obtained cDNAs revealed that none of the $n d h \mathrm{~B}$ or $y c f 3$ editing sites were edited in mitochondria (see Fig. 1C,D for chromatograms). Notably, there is not even a trace of editing detectable. To exclude the possibility that RNA editing is impaired in unspliced transcripts due to the presence of the intron, vector pHS587 was constructed (see Fig. 1A) containing the $n d h \mathrm{~B}$ sequence without intron. However, transcripts of pHS587 were also found not to be edited in maize mitochondria (not shown), indicating that removal of the intron is not sufficient to trigger RNA editing of the plastid transcript. However, editing and splicing may be linked processes, where the unprocessed pre-mRNA may have to be integrated into a processing complex, as previously suggested by Choury et al. (2005). For this reason, construct pHS588 (see Fig. 1A) was established, exchanging the plastid $n d h \mathrm{~B}$ intron with the mitochondrial cox 2 intron from maize. However, RT-PCR failed to detect transcripts of pHS588 in maize and cauliflower mitochondria. The fact that, in this study, no transcripts, not even unspliced RNA, were detectable, is similar to results from Holländer and Kück (1999), where an impaired tertiary intron structure led to a complete loss of exon RNA.

It has been suggested that there are specific factors for each of the $\sim 30$ plastid editing sites (Chaudhuri et al. 1995; Hirose and Sugiura 2001; Miyamoto et al. 2004; Okuda et al. 2006). One protein family that is a candidate for housing such factors is the PPR protein family, for which there are literally hundreds of members in plants with many having known targeting sites, thereby implying a possible role in RNA processing - at least for some of the members. Two editing site-specific factors are known for plastid RNA editing in A. thaliana, the PPR proteins CRR4 and CRR21, whereas CRR4 is shown to be exclusively localized in plastids (Kotera et al. 2005; Okuda et al. 2006, 2007). In A. thaliana, 442 PPR proteins are known, approximately a quarter of which have a plastid targeting site. Although they certainly may have other functions than RNA editing (for review, see Andrés et al. 2007), there is a sufficiently high number available to match the number of plastid RNA editing sites. In contrast, there are up to 500 RNA editing sites in higher plant mitochondria (summarized in Mower and Palmer 2006). This large number of editing sites certainly exceeds the number of PPR proteins with predicted mitochondrial localization, raising the question if plastid and mitochondrial RNA editing site recognition work in the same way. Indeed, in the last decade, Sutton et al. (1995) transformed a mitochondrial exon (petunia hybrida cox2) into tobacco plastids, which were not edited. In addition, a transcript of mitochondrial localized rpoB fragment of plastid origin was found not to be edited in rice (Zeltz et al. 1996). Similarly, in our approach, we found that transcripts of the plastid genes are not edited in plant mitochondria. This may be due to the fact that plastid targeted editing site-specific factors are not present in plant mitochondria.

Editing systems of mitochondria and plastids display many similarities, as already pointed out by Tillich et al. (2006). The similarities include the location and extent of the cis-elements required for RNA editing. These cis-elements are discussed as potential binding sites for trans-acting factors that guide the editing enzyme(s) to the respective site. Thus, we were interested in a sequence comparison between the maize chloroplast $n d h \mathrm{~B}$ editing sites and the mitochondrial editing sites from maize. As determined in many approaches (for review, see Shikanai 2006), a 20-nucleotide (nt) sequence upstream of the editing site is generally required for recognition of the site and, in some cases, also $\sim 10 \mathrm{nt}$ downstream. We therefore chose both the upstream and downstream regions for sequence comparison. The sequences of the six $Z$. mays $n d h \mathrm{~B}$ editing sites were compared to those of all known maize mitochondrial editing sites in the NCBI database. As shown in Figure 2, three $n d h \mathrm{~B}$ editing sites exhibit considerable sequence similarities with maize mitochondrial editing sites. This is particularly true for nucleotides directly upstream of the editing sites; however, in some cases, similarities are also found further upstream. If there are site-specific RNA editing factors present in plant mitochondria, the obvious difference in the number of editing sites and PPR proteins available would require each PPR protein to recognize several RNA editing sites with similar sequences. Indeed, Choury et al. (2004) have shown that RNA editing site recognition tolerates point mutations at most positions in the cis-elements, although this reduces RNA editing efficiency. However, despite there being considerable sequence similarity between maize $n d h \mathrm{~B}$ and maize mitochondrial editing sites, we found no evidence of RNA editing of $n d h \mathrm{~B}$ transcripts in our in organello system. Unlike for plastid RNA editing, there is no evidence to suggest that site-specific factors are involved in mitochondrial RNA editing. In a previous paper, we proposed a self-guiding-transcript model for mitochondrial RNA editing, where the secondary or tertiary structure of a transcript contains the editing information without requirement for 


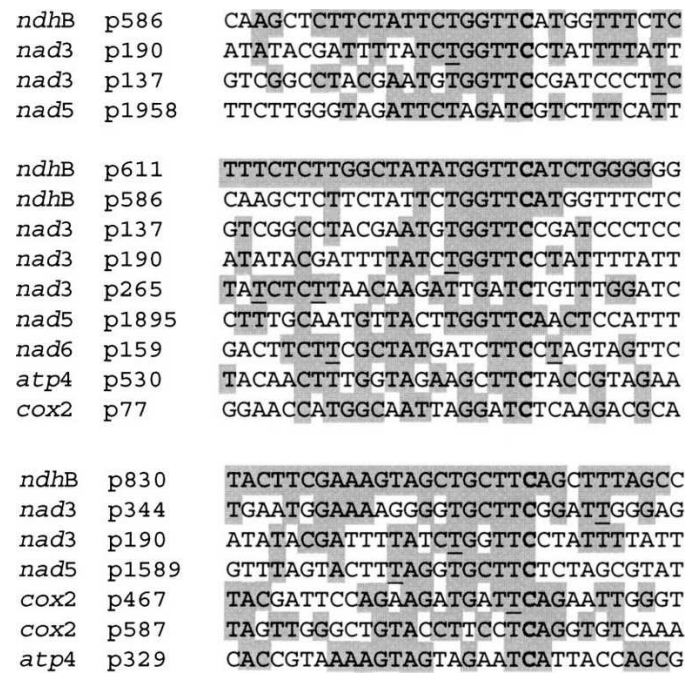

FIGURE 2. Alignment of sequences surrounding three $Z$. mays $n d h \mathrm{~B}$ editing sites and $Z$. mays mitochondrial editing sites. The maize $n d h \mathrm{~B}$ editing sites were first identified by Maier et al. (1992) and the mitochondrial editing sites shown here are from the NCBI database (accession no. AY506529). The alignments were made with a total of 271 editing sites from maize mitochondrial transcripts and the sequences with the best matches are shown here. The editing sites were numbered according to the appropriate ORF. Nucleotides identical to the $n d h \mathrm{~B}$ sequences are highlighted in gray block. Editing positions are shown in bold letters. In some cases, editing positions within the surrounding sequences have been changed from $\mathrm{C}$ to $\mathrm{T}$ for better alignment. These positions are underlined.

site-specific factors. If this holds true, as suggested by the analysis of the data presented here, the RNA editing site recognition in higher plant mitochondria and plastids is indeed different.

\section{MATERIALS AND METHODS}

\section{Plant lines used}

Maize (line Kampala, Nickerson SA) mitochondria were isolated from 12-d-old etiolated seedlings as described in Newton (1994). Cauliflower heads were obtained from local grocery stores.

\section{Mitochondrial electroporation and in organello incubation}

All mitochondrial procedures were performed as described (Staudinger and Kempken 2003; Kempken et al. 2007), with the exception of $1200 \mu \mathrm{g}$ mitochondrial protein used. In organello incubation of mitochondria was carried out as previously described for $3 \mathrm{~h}$.

\section{Nucleic acid isolation and gel electrophoresis}

Mitochondrial nucleic acids were isolated as described (Staudinger and Kempken 2003). Bacterial plasmid DNA was isolated using either a NucleoBond Reagent Set (Macherey-Nagel) or the Plasmid Midi Kit (QIAGEN).

\section{PCR, RT-PCR, and sequence analysis}

PCR, RT-PCR, and sequence analyses were done as described previously (Bolle and Kempken 2006).

\section{Vector construction}

The vectors pTJ246 and pNB475, containing the cox 2 gene from $A$. thaliana and the cox2-coding sequence including the intron from maize, respectively, were described in previous studies (Staudinger and Kempken 2003; Bolle and Kempken 2006).

The vectors pCW399 and pCW407 contain the 5' and 3' untranslated regions of the $Z$. mays atp 6 gene, amplified by PCR with primer pairs FK433 (5'-GGGGGATCCCCAACTCTACC CACGCTTCGG-3')/FK434 (5'-GGGGATATCGTACAGATCTCT TAGAGGCTG- $\left.3^{\prime}\right)$ and FK435 (5'-GGGAAGCTTTAGCATTAAC CGGTCTGGAATTAG-3')/FK436 (5'-GGGGGATCCAACACAA GCAAGTTGAACTTAC- $3^{\prime}$ ). In vector pNB569, the $5^{\prime}$ and $3^{\prime}$ untranslated regions are from the $A$. thaliana cox 2 gene, obtained by PCR using primer pairs FK351 (5'-GGGGGGGCGGCCGCG CCTTACCACACCAACCACCGGGGGGGCGGCCGCGCCTTAC CACACCAACCACC-3')/FK639 (5'-GATATCTTTCAAAGCAAA GGTTCCTTC-3') and FK640 (5'-GATATCGCGGAAATTTAA GAGTAAGGTG-3')/FK641 (5'-CCCGGGCCTGAGGCAAAGGC GAATC-3'). The amplicons were cloned in pBluescript SK+ (Stratagene). The $Z$. mays $n d h B$-coding sequence including intron was amplified using primer pair FK618 (5'-GGGATGATCTGG CATGTACAGAA-3')/FK624 (5'-GAAGCTTCTAAAAGAGGGT ATCCTGAG- $3^{\prime}$ ) and cloned between the $5^{\prime}$ and $3^{\prime}$ untranslated regions in pCW399 and pNB569. The Z. mays ycf3-coding sequence including the introns was amplified using primer pair FK668 (5'-GGGATGCCTAGATCCCG-3')/FK670 (5'-AAGCTTT TATTCAAATTCAAAGC-3') and cloned between the $Z$. mays atp6 $5^{\prime}$ and $3^{\prime}$ untranslated regions in pCW407. The $y c f 3$ ORF is shortened by 116 bp of the $3^{\prime}$ region and this region is replaced by the corresponding $Z$. mays atp6 region. Since both plasmids pHS587 and pHS588 are based on pNB569, an inverse PCR was performed using oligonucleotides NB923 (5'-TCCTTCGTAG ACGTCAGGAGTC-3') and NB924 (5'-TCCCCCACTCCAGTCG TTG-3'), amplifying the whole sequence of pNB569 except the $n d h \mathrm{~B}$ intron. To generate construct pHS587, this amplicon was ligated. To generate construct pHS588, this amplicon was ligated with the cox 2 intron from $Z$. mays, obtained by PCR using oligonucleotides NB921 (5'-GTGCGCCTCTTAACGAGGGTG-3') and NB922 (5'-GTTGGGTCGACGCCCGCC-3'). Oligonucleotides were derived from the published sequence of the mitochondrial DNA of A. thaliana (NCBI accession no. NC001284) and Z. mays (NCBI accession no. AY506529), as well as from the published sequence of the chloroplast DNA of $Z$. mays (NCBI accession no. NC_001666). All oligonucleotides used for cloning of pCW399, pNB569, and pCW407 contained additional sequences to allow recovery of subcloned PCR fragments by restriction digest. Vectors and localization of oligonucleotides used in RT-PCR are shown in Figure 1A.

\section{Standard procedures}

All other standard molecular biology techniques were performed according to standard published procedures (Sambrook et al. 1989). 


\section{ACKNOWLEDGMENTS}

We thank Mrs. Hanna Schmidt for excellent technical assistance. We are grateful to Mrs. Christina Wehling for constructing vectors pCW399 and pCW407. This work was supported by a grant from the DFG (Ke409/15-2).

Received July 31, 2007; accepted September 11, 2007.

\section{REFERENCES}

Andrés, C., Lurin, C., and Small, I.D. 2007. The multifarious roles of PPR proteins in plant mitochondrial gene expression. Physiol. Plant. 129: 14-22.

Bolle, N. and Kempken, F. 2006. Mono- and dicotyledonous plantspecific RNA editing sites are correctly edited in both in organello systems. FEBS Lett. 580: 4443-4448.

Chaudhuri, S., Carrer, H., and Maliga, P. 1995. Site-specific factor involved in the editing of the $p s b L$ mRNA in tobacco plastids. EMBO J. 14: 2951-2957.

Choury, D., Farre, J.C., Jordana, X., and Araya, A. 2004. Different patterns in the recognition of editing sites in plant mitochondria. Nucleic Acids Res. 32: 6397-6406. doi: 10.1093/nar/gkh969.

Choury, D., Farré, J.C., Jordana, X., and Araya, A. 2005. Gene expression studies in isolated mitochondria: Solanum tuberosum rps10 is recognized by cognate potato but not by the transcription, splicing and editing machinery of wheat mitochondria. Nucleic Acids Res. 33: 7058-7065. doi: 10.1093/nar/gki1017.

Clifton, S.W., Minx, P., Fauron, C.M., Gibson, M., Allen, J.O., Sun, H., Thompson, M., Barbazuk, W.B., Kanuganti, S., Tayloe, C., et al. 2004. Sequence and comparative analysis of the maize NB mitochondrial genome. Plant Physiol. 136: 34863503.

Herdenberger, F., Holländer, V., and Kück, U. 1994. Correct in vivo RNA splicing of a mitochondrial intron in algal chloroplasts. Nucleic Acids Res. 22: 2869-2875. doi: 10.1093/nar/22.15.2869.

Hirose, T. and Sugiura, M. 2001. Involvement of a site-specific transacting factor and a common RNA-binding protein in the editing of chloroplast mRNAs: Development of a chloroplast in vitro RNA editing system. EMBO J. 20: 1144-1152.

Holländer, V. and Kück, U. 1999. Group II intron splicing in chloroplasts: Identification of mutations determining intron stability and fate of exon RNA. Nucleic Acids Res. 27: 2345-2353. doi: 10.1093/nar/27.11.2345.

Jenkins, B.D. and Barkan, A. 2001. Recruitment of a peptidyl-tRNA hydrolase as a facilitator of group II intron splicing in chloroplasts. EMBO J. 20: 872-879.

Jenkins, B.D., Kulhanek, D.J., and Barkan, A. 1997. Nuclear mutations that block group II RNA splicing in maize chloroplasts reveal several intron classes with distinct requirements for splicing factors. Plant Cell 9: 283-296.

Kempken, F., Bolle, N., Forner, J., and Binder, S. 2007. Transcript end mapping and analysis of RNA editing in plant mitochondria. In Methods Mol. Biol.: Mitochondria (eds. D. Leister and J.M. Herrmann), pp. 177-192. Humana Press, Totowa, NJ.
Kotera, E., Tasaka, M., and Shikanai, T. 2005. A pentatricopeptide repeat protein is essential for RNA editing in chloroplasts. Nature 433: $326-330$.

Maier, R., Neckermann, K., Hoch, B., Akhmedov, N.B., and Kössel, H. 1992. Identification of editing positions in the $n d h B$ transcript from maize chloroplasts reveals sequence similarities between editing sites of chloroplasts and plant mitochondria. Nucleic Acids Res. 20: 6189-6194. doi: 10.1093/nar/20.23.6189.

Miyamoto, T., Obokata, J., and Sugiura, M. 2004. A site-specific factor interacts directly with its cognate RNA editing site in chloroplast transcripts. Proc. Natl. Acad. Sci. 101: 48-52.

Mower, J.P. and Palmer, J.D. 2006. Patterns of partial RNA editing in mitochondrial genes of Beta vulgaris. Mol. Genet. Genomics 276: 285-293.

Newton, K.J. 1994. Procedures for isolating mitochondria and mitochondrial DNA and RNA. In The maize handbook (eds. M. Freeling and V. Walbot), pp. 549-556. Springer, New York.

Okuda, K., Nakamura, T., Sugita, M., Shimizu, T., and Shikanai, T. 2006. A pentatricopeptide repeat protein is a site recognition factor in chloroplast RNA editing. J. Biol. Chem. 281: 37661-37667.

Okuda, K., Myouga, F., Motohashi, R., Shinozaki, K., and Shikanai, T. 2007. Conserved domain structure of pentatricopeptide repeat proteins involved in chloroplast RNA editing. Proc. Natl. Acad. Sci. 104: $8178-8183$.

Ostheimer, G.J., Williams-Carrier, R., Belcher, S., Osborne, E., Gierke, J., and Barkan, A. 2003. Group II intron splicing factors derived by diversification of an ancient RNA-binding domain. EMBO J. 22: 3919-3929.

Peeters, N.M. and Hanson, M.R. 2002. Transcript abundance supercedes editing efficiency as a factor in developmental variation of chloroplast gene expression. RNA 8: 497-511.

Sambrook, J., Fritsch, E.F., and Maniatis, T. 1989. Molecular cloning: A laboratory manual. Cold Spring Harbor Laboratory Press, Cold Spring Harbor, NY.

Shikanai, T. 2006. RNA editing in plant organelles: Machinery, physiological function and evolution. Cell. Mol. Life Sci. 63: 698-708.

Staudinger, M. and Kempken, F. 2003. Electroporation of higherplant mitochondria: Transcripts of an introduced $\operatorname{cox} 2$ gene, but not an atp6 gene, are edited in organello. Mol. Genet. Genomics 269: $553-561$.

Stern, D.B. and Lonsdale, D.M. 1982. Mitochondrial and chloroplast genomes of maize have a 12-kilobase DNA sequence in common. Nature 229: 698-702.

Sutton, C.A., Zoubenko, O.V., Hanson, M.R., and Maliga, P. 1995. A plant mitochondrial sequence transcribed in transgenic tobacco chloroplasts is not edited. Mol. Cell. Biol. 15: 1377-1381.

Tillich, M., Lehwark, P., Morton, B.R., and Maier, U.G. 2006. The evolution of chloroplast RNA editing. Mol. Biol. Evol. 23: 1912-1921.

Zeltz, P., Kadowaki, K.I., Kubo, N., Maier, R.M., Hirai, A., and Kössel, H. 1996. A promiscuous chloroplast DNA fragment is transcribed in plant mitochondria but the encoded RNA is not edited. Plant Mol. Biol. 31: 647-656.

Zeng, W.H., Liao, S.C., and Chang, C.C. 2007. Identification of RNA editing sites in chloroplast transcripts of Phalaenopsis aphrodite and comparative analysis with those of other seed plants. Plant Cell Physiol. 48: 362-368. 

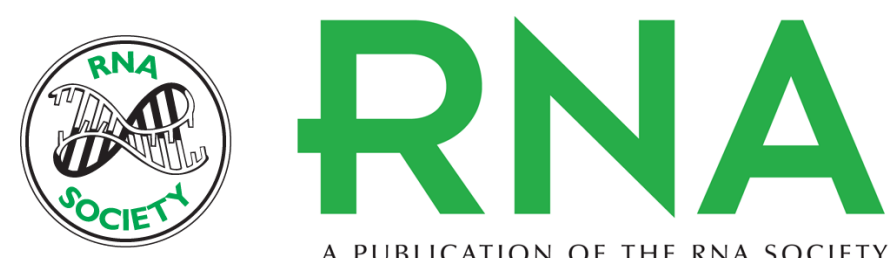

A PUBLICATION OF THE RNA SOCIETY

\section{Plastid mRNAs are neither spliced nor edited in maize and cauliflower mitochondrial in organello systems}

Nina Bolle, Inga Hinrichsen and Frank Kempken

RNA 2007 13: 2061-2065

References This article cites 26 articles, 10 of which can be accessed free at:

http://rnajournal.cshlp.org/content/13/12/2061.full.html\#ref-list-1

\section{License}

Email Alerting Receive free email alerts when new articles cite this article - sign up in the box at the Service top right corner of the article or click here. 